\title{
Utilização da Simulação de Processo para Prever o Comportamento do Sistema de Tratamento de Efluente Ácido em uma Indústria de Cloro Soda
}

\author{
J. Z. A. PAFFER ${ }^{1}$, J. I. H. T. NETO ${ }^{2}$, L. S. PEDROSA ${ }^{2}$, R. BRITO ${ }^{1}$ e A. B. ATHANASAKIS ${ }^{1}$ \\ ${ }^{1}$ Universidade Federal de Campina Grande, Unidade Acadêmica de Engenharia Química \\ ${ }^{2}$ Braskem S. A., Departamento de Engenharia de Processos \\ E-mail para contato: juzeymer@yahoo.com.br
}

\begin{abstract}
RESUMO - Um dos efluentes gerados na indústria de cloro e soda é uma corrente ácida (água saturada de cloro). Esta corrente deve passar por um sistema de tratamento antes de ser descartado, devido ao potencial de dano que este pode causar à flora e à fauna marinha, além de estar fora das especificações ambientais. O domínio do comportamento deste sistema de tratamento é importante na gestão ambiental e operacional da indústria de cloro soda. Uma forma muito utilizada para estudar o comportamento de sistemas, é a simulação de processos. Com esta é possível, através de distúrbios inseridos no sistema, conhecer a dinâmica do sistema, inclusive prescindindo de teste de campo, muitas vezes impossíveis de realização seja por questão de segurança ou econômica. A simulação deste sistema possibilita determinar as condições de controle, visando otimizar a operação, reduzindo o consumo de vapor e eliminando a concentração de cloro na água.
\end{abstract}

\section{INTRODUÇÃO}

A importância da indústria de cloro e soda no mundo é indiscutível. Estes produtos estão entre os dez químicos mais produzidos no mundo e estão ligados à maioria da produção de outros produtos químicos que são utilizados no dia a dia da sociedade (O’Brien at al., 2005).

As aplicações do cloro são diversas e variam desde a produção do gás lacrimogênio até o tratamento de água. Os principais segmentos do mercado mundial que consomem cloro são vinílicos (33\%) e produtos orgânicos (19\%). Já o mercado de soda está dividido principalmente entre produtos orgânicos (18\%), papel e celulose (16\%) e produtos inorgânicos (15\%) (Junior, 2006).

Atualmente, o Brasil tem uma demanda de soda cáustica maior do que a oferta interna, o que faz com que 39,47\% do consumo interno sejam atendidos por importações (Nogueira, 2011). Este cenário faz com que as indústrias brasileiras de cloro e soda busquem continuamente maior competitividade no mercado através de várias frentes, onde uma delas é a otimização de processos. Esta otimização busca a redução de custos, aumento da produção e da qualidade através de melhores condições de operação de processos.

Sabendo que as condições de processo da planta não são constantes, variáveis de controle 
podem ser alteradas através de controladores automáticos e na atuação dos operadores. Com o objetivo de conhecer o comportamento dinâmico do processo, a simulação computacional é muito utilizada para auxiliar no projeto de controladores e estudar comportamentos do sistema (Stephanopoulos, 1938).

Uma ferramenta muito importante utilizada hoje para estudar comportamentos de sistemas é a simulação de processos. Com esta é possível determinar e avaliar tanto variáveis dificilmente medidas, como as internas do processo, como variáveis mensuráveis, possibilitando maior compreensão dos fenômenos físicos do sistema. Também é possível realizar distúrbios no sistema que dificilmente seriam realizadas no campo, tanto por fatores de segurança como econômicos (Maneguelo, 2007).

\subsection{Processo Cloro Soda}

De acordo com Hine (1985), o processo de produção industrial de cloro e soda de tecnologia de diafragma consiste basicamente em uma área de tratamento de salmoura, eletrólise, evaporação da soda e processamento de cloro e hidrogênio, no entanto na unidade da BRASKEM em Alagoas contém também o processo de mineração e produção de dicloroetano (DCE), conforme a Figura 1.

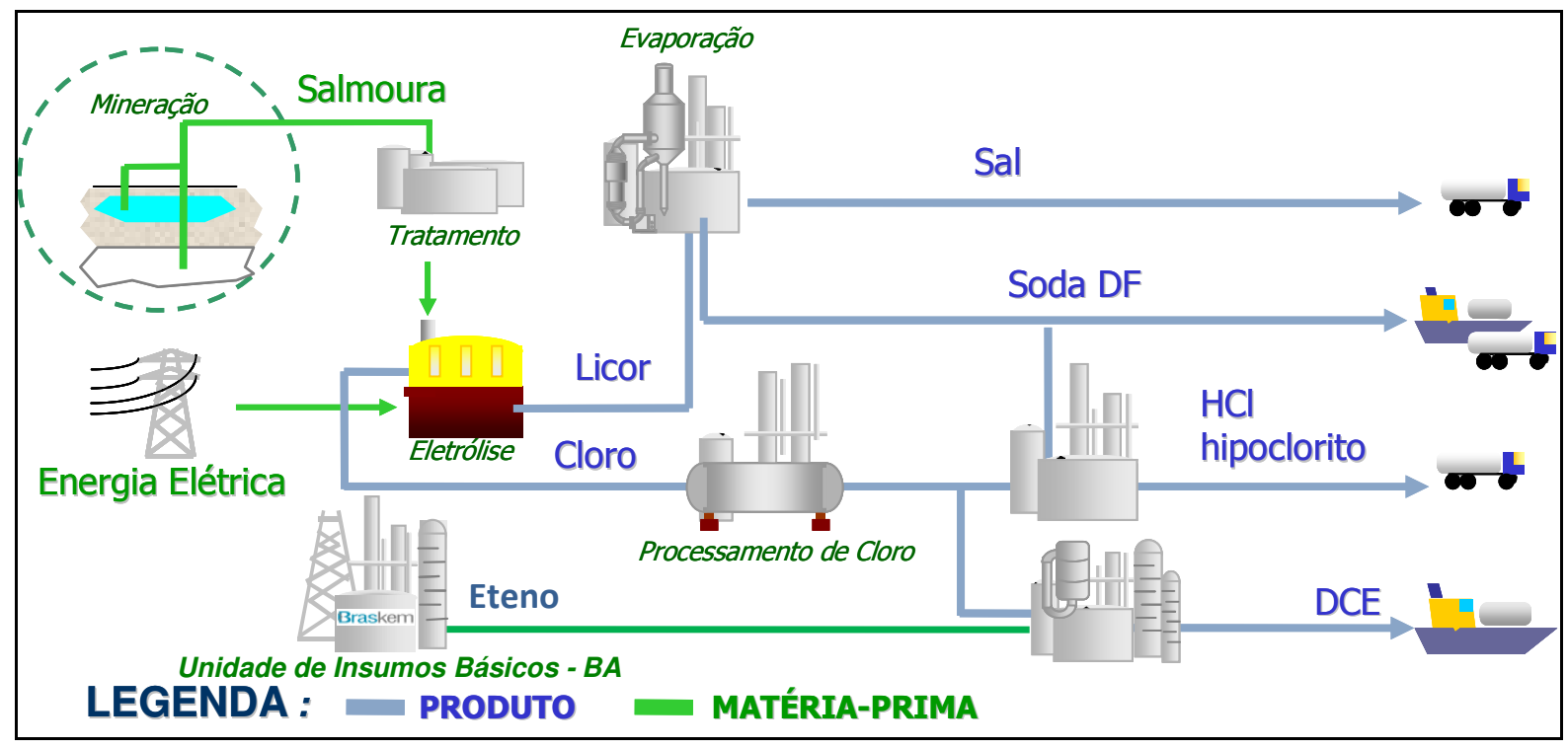

Figura 1: Desenho simplificado do processo de uma indústria cloro e soda de tecnologia diafragma da BRASKEM.

A solução aquosa de sal a uma concentração de $320 \mathrm{~g} / \mathrm{l}$ de $\mathrm{NaCl}$, também conhecida como salmoura, é obtida na mineração e encaminhada para o tratamento. Em seguida é filtrada, aquecida, ressaturada e enviada para a eletrólise.

Esta salmoura tratada é injetada na célula onde ocorre a eletrólise, segundo a Equação 1. 


\section{9 a 22 de outubro de 2014 \\ Florianópolis/SC}

$$
\begin{array}{lcccc}
2 \mathrm{NaCl}_{(s o l) l}+ & 2 \mathrm{H}_{2} \mathrm{O}_{(l)} & \longrightarrow & 2 \mathrm{NaOH}_{(s o l) l}+\mathrm{Cl}_{2(g)}+H_{2(g)} \\
\text { Sal Água } & & \text { Soda Cloro Hidrogênio }
\end{array}
$$

Após esta etapa o cloro segue para a área de processamento com o objetivo de retirar as impurezas, através do resfriamento e liquefação, para posterior envio para a área de produção de dicloroetano. Já o hidrogênio é distribuído para as caldeiras e para a área de produção de ácido clorídrico e aquecimento da salmoura. A solução aquosa composta por $\mathrm{NaOH}(12 \%$ em massa) e $\mathrm{NaCl}$ (17\% em massa), também conhecida como licor de células, flui por gravidade para o tanque de licor e posterior envio para a evaporação até a concentração da soda em 50\%.

O processamento de cloro tem como objetivo retirar impurezas raro hidrogênio, nitrogênio, oxigênio, dióxido de carbono, água e sal para a sua possível utilização na produção de DCE. O sistema de processamento de cloro abrange as etapas de resfriamento, filtração, secagem, compressão, liquefação e estocagem/abatimento.

O processo de resfriamento de cloro visa eliminar $99 \%$ da água que foi arrastada na forma de vapor da eletrólise. Através de dois trocadores de calor, onde o primeiro utiliza água da torre como líquido refrigerante e o segundo utiliza água gelada. A temperatura limite que o cloro pode atingir no resfriamento é de $10^{\circ} \mathrm{C}$, pois abaixo desta temperatura há a formação de cristais de cloro que dificultam a passagem do gás. No final do processo de resfriamento há um filtro de cloro úmido com o objetivo de reter o teor residual de cloreto de sódio que pode ser arrastado com o cloro gás.

A água condensada nos dois estágios de resfriamento é coletada num vaso de água clorada, onde em seguida uma parte é bombeada para a cloração da salmoura com a finalidade de eliminar a amônia, e outra para a torre de stripper que tem a finalidade de retirar o cloro da água (Costa, 2004).

\subsection{Processo de Tratamento do Efluente Ácido}

A finalidade da torre de stripper da área de cloro é tratar o efluente ácido, ou seja, eliminar o cloro contido na água retirada do sistema, nos estágios de resfriamento e filtragem tornando-a inofensiva ao meio ambiente. A água com cloro dissolvido segue o equilíbrio da Equação 2.

$$
\begin{aligned}
& \mathrm{Cl}_{2(\text { sol })}+\mathrm{H}_{2} \mathrm{O}_{(l)} \longleftrightarrow \mathrm{HOCl}_{(l)}+\mathrm{HCl}_{(l)} \\
& \text { Cloro Água Ácido Hipocloros o Ácido Clorídrico }
\end{aligned}
$$

Com o objetivo de deslocar o equilíbrio da Equação 2 para a esquerda, é injetado $\mathrm{HCl}$ na torre de stripper de 22 à $25 \%$ de concentração. Desta forma, ao fornecer íons $\mathrm{H}^{+}$, há a decomposição da parte do cloro que reagiu com a água retornando à forma de $\mathrm{Cl}_{2}$.

Com a elevação da temperatura da água clorada próximo ao ponto de ebulição $\left(99,4{ }^{\circ} \mathrm{C}\right)$, através de pré-aquecimento da água clorada e do contato direto com o vapor, o cloro desprende-se da água e sai pela parte superior da torre de stripper, onde é enviada para a entrada do resfriador 
primário. Já a água praticamente livre de cloro sai pelo fundo da torre onde é enviada diretamente para a área de tratamento de efluentes.

O presente trabalho possui forte motivação de aspectos econômicos e ambientais por possibilitar a redução de custos e maior controle na qualidade do efluente ao otimizar o processo de stripping de água clorada. A estimativa de redução de custo operacional baseia-se na otimização do consumo de vapor. Com o processo de stripping de cloro ocorrendo de modo otimizado, é possível eliminar a concentração de cloro na corrente de água com o consumo mínimo de vapor. Desta forma, além da redução do consumo de vapor, reduz também o envio de vapor junto com a corrente de cloro para o sistema de resfriamento e um possível envio de cloro para o efluente.

\section{METODOLOGIA}

A mistura do sistema de objeto de estudo, água clorada, contém na sua solução ácido clorídrico e água, que se caracteriza como uma solução eletrolítica, uma vez que há a dissociação parcial destes componentes, conforme a Equação 3 (Aspen, 2011).

$$
\mathrm{HCl}+\mathrm{H}_{2} \mathrm{O} \leftarrow \rightarrow \mathrm{H}_{3} \mathrm{O}^{+}+\mathrm{Cl}^{-}
$$

Com isto, devido a presença de eletrólitos, o simulador escolhido foi o Aspen ${ }^{\mathrm{TM}}$, uma vez que o Hysys não possui modelos termodinâmicos para estes casos. Entre os modelos termodinâmicos, o que é capaz de prever o comportamento de eletrólitos no Aspen é o Electrolyte NRTL (ElecNRTL) (LIN et al., 2009).

As etapas seguidas para a simulação em regime estacionário foram:

- Conhecer o objeto de estudo;

- Determinar o modelo termodinâmico a ser utilizado;

- Determinar as correntes de entrada e saída do sistema;

- Desenhar o sistema no Aspen ${ }^{\mathrm{TM}}$;

- Convergir a simulação.

\section{RESULTADOS E DISCUSSÕES}

A simulação do sistema compreende a torre de stripper, a bomba de retirada da água declorada e o dessuperaquecimento do vapor antes da entrada na torre, conforme a Figura 2. 


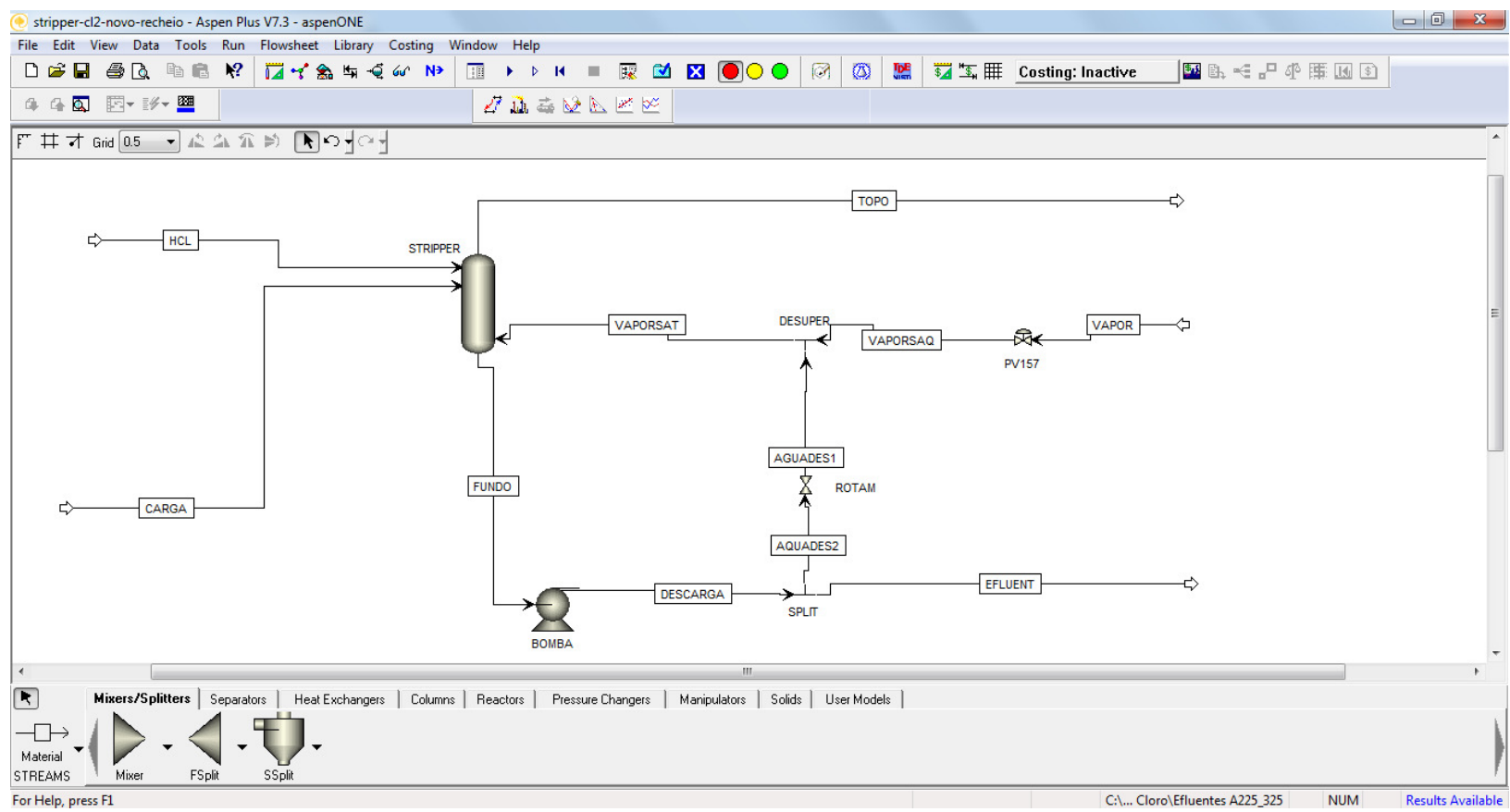

Figura 2: Tela da simulação da torre de stripper de cloro objeto de estudo em regime estacionário no Aspen ${ }^{\mathrm{TM}}$.

As correntes de entrada e saída do sistema são:

- $\mathrm{HCl}: \mathrm{HCl}$ à 24\% de concentração;

- CARGA: água clorada do sistema do vaso de água clorada;

- VAPORSAT: vapor saturado;

- VAPORSAQ: vapor superaquecido da área de utilidades;

- TOPO: cloro gás desprendido da água clorada e vapor de água;

- FUNDO: água praticamente livre de cloro, água declorada.

A Tabela 1 apresenta as especificações das correntes de entrada. 
Tabela 1 - Especificações das correntes de entrada da torre de stripper.

\begin{tabular}{|c|c|c|c|c|c|c|}
\hline & \multicolumn{3}{|c|}{ Composição - fração molar } & \multirow{2}{*}{$\begin{array}{c}\text { Temperatura } \\
\left({ }^{\circ} \mathrm{C}\right)\end{array}$} & \multirow{2}{*}{$\begin{array}{l}\text { Pressão } \\
\left(\mathrm{kg} / \mathrm{cm}^{2}\right)\end{array}$} & \multirow{2}{*}{$\begin{array}{c}\text { Vazão mássica } \\
(\mathrm{kg} / \mathrm{hr})\end{array}$} \\
\hline Corrente & Cloro & Água & $\mathrm{HCl}$ & & & \\
\hline $\mathrm{HCL}$ & - & 0,76 & 0,24 & 30 & 2 & 779,5 \\
\hline CARGA & 0,0047523 & 0,9952477 & - & 40 & 1,5 & 40191 \\
\hline VAPORSAQ & - & 1 & - & 156,766 & 1,05 & 4521,376 \\
\hline VAPORSAT & $9,53 \times 10^{-9}$ & 0,9999231 & $7,69 \times 10^{-5}$ & 111,433 & 0,5 & 4697,676 \\
\hline
\end{tabular}

Com estas especificações, as correntes de $\mathrm{HCl}$ e CARGA (água clorada) entram pela parte superior da torre de stripper e a corrente VAPORSAT (vapor saturado) entra na parte inferior. Após alguns ajustes, conseguiu-se que a simulação convergisse, onde foi possível obter os resultados das correntes de saída, conforme a Tabela 2.

Tabela 2: Especificações das correntes de saída da torre de stripper.

\begin{tabular}{|l|c|c|c|c|c|c|}
\cline { 2 - 4 } \multicolumn{1}{c|}{} & \multicolumn{2}{c|}{ Composição - fração molar } & \multirow{2}{*}{$\begin{array}{c}\text { Temperatura } \\
\left({ }^{\circ} \mathbf{C}\right)\end{array}$} & $\begin{array}{c}\text { Pressão } \\
\left(\mathbf{k g} / \mathbf{c m}^{\mathbf{2}}\right)\end{array}$ & $\begin{array}{c}\text { Vazão mássica } \\
(\mathbf{k g} / \mathbf{h r})\end{array}$ \\
\hline TOrrente & Cloro & Água & $\mathbf{H C l}$ & 94,293 & 0,0025 & 396,82 \\
\hline FUNDO & 0,1907235 & 0,8092748 & $1,66 \times 10^{-6}$ & 94 & 45272 \\
\hline
\end{tabular}

Com estes resultados é possível observar que a corrente de FUNDO sai da torre de stripper praticamente livre de cloro, o que já satisfaz a legislação ambiental. Já a corrente de TOPO sai da torre de stripper com uma concentração significativa de cloro que retornará para o processo na entrada do resfriador primário.

\section{CONCLUSÕES}

Com estes resultados, pode-se chegar a conclusão que a simulação em regime estacionário convergiu e que pode ser utilizada para simular diversas situações que ocorrem no dia a dia da planta, devido a pressão do sistema ser igual a pressão resultante da simulação. Esta ferramenta é muito útil para aumentar o conhecimento do sistema com a determinação de variáveis dificilmente medidas possibilitando maior compreensão dos fenômenos físicos do sistema.

Uma sugestão para trabalhos futuros é a simulação dinâmica deste processo onde é possível realizar distúrbios no sistema que dificilmente seriam realizadas no campo, tanto por fatores de segurança como econômicos.

\section{REFERÊNCIAS}

ASPENTM V7.3. Help. Aspen Technology, 2011. 
COSTA, C. A. Monografias de processo da área de cloro. BRASKEM S.A. Maceió, 2004.

HINE, F. Electrode Processes and Electrochemical Engeneering. Plenum Press. New York, 1985.

JUNIOR, R. N. J. Modelagem Matemática de um Processo Industrial de Produção de Cloro e Soda por Eletrólise de Salmoura Visando a sua Otimização. Escola Politécnica da Universidade de São Paulo. Departamento de Engenharia Química. São Paulo, 2006.

LIN, Y.; KATE, A. T.; MOOIJER, M. et al. Comparison of activity coefficient models for electrolyte system. AlChE Journal, p n/a-n/a, 2009.

MANEGUELO, A. P. Contribuições à análise e modelagem de operações transientes de colunas de destilação. Universidade Federal de Santa Catarina. Florianópolis, 2007.

NOGUEIRA, A. H. Perfil da Indústria Brasileira de Cloro e Soda. INDI, Instituto de Desenvolvimento Integrado de Minas Gerais. Brasil, 2011.

O'BRIEN, T. F.; BOMMARAJU, T. V.; HINE, F. Handbook of Chlor-Alkali Technology. Springer, New York, 2005.

STEPHANOPOUlOS, G. Chemical Process Control: An Introduction to Theory and Practice. Prantice Hall. Englewood Cliffs, New Jersey, 1983. 